\title{
Differences in arachidonic acid levels and fatty acid desaturase (FADS) gene variants in African Americans and European Americans with diabetes or the metabolic syndrome
}

\author{
Susan Sergeant ${ }^{1,2}$, Christina E. Hugenschmidt ${ }^{3}$, Megan E. Rudock ${ }^{4}$, Julie T. Ziegler ${ }^{5}$, Priscilla Ivester ${ }^{1,6}$, \\ Hannah C. Ainsworth ${ }^{1,6}$, Dhananjay Vaidya ${ }^{7}$, L. Douglas Case ${ }^{5}$, Carl D. Langefeld ${ }^{5}$, Barry I. Freedman ${ }^{8}$, \\ Donald W. Bowden ${ }^{2,3,4}$, Rasika A. Mathias ${ }^{7,9}$ and Floyd H. Chilton ${ }^{1,6 *}$ \\ ${ }^{1}$ The Center for Botanical Lipids and Inflammatory Disease Prevention, Wake Forest School of Medicine, Winston-Salem, \\ NC 27157, USA \\ ${ }^{2}$ Department of Biochemistry, Wake Forest School of Medicine, Winston-Salem, NC 27157, USA \\ ${ }^{3}$ Center for Diabetes Research, Wake Forest School of Medicine, Winston-Salem, NC 27157, USA \\ ${ }^{4}$ Center for Human Genomics, Wake Forest School of Medicine, Winston-Salem, NC 27157, USA \\ ${ }^{5}$ Division of Public Health Sciences, Department of Biostatistical Sciences, Wake Forest School of Medicine, Winston-Salem, \\ NC 27157, USA \\ ${ }^{6}$ Department of Physiology/Pharmacology, Wake Forest School of Medicine, Winston-Salem, NC 27157, USA \\ ${ }^{7}$ Division of General Internal Medicine, Department of Medicine, The Johns Hopkins University, Baltimore, MD 21224, USA \\ ${ }^{8}$ Department of Internal Medicine, Wake Forest School of Medicine, Winston-Salem, NC 27157, USA \\ ${ }^{9}$ Division of Allergy and Clinical Immunology, Department of Medicine, The Johns Hopkins University, Baltimore, \\ MD 21224, USA \\ (Received 10 December 2010 - Revised 11 April 2011 - Accepted 5 May 2011 - First published online 4 July 2011)
}

\section{Abstract}

Over the past 50 years, increases in dietary $n$ - 6 PUFA, such as linoleic acid, have been hypothesised to cause or exacerbate chronic inflammatory diseases. The present study examines an individual's innate capacity to synthesise $n$ - 6 long-chain PUFA (LC-PUFA) with respect to the fatty acid desaturase (FADS) locus in Americans of African and European descent with diabetes or the metabolic syndrome. Compared with European Americans (EAm), African Americans (AfAm) exhibited markedly higher serum levels of arachidonic acid (AA) (EAm 7.9 (SD 2.1), AfAm $9 \cdot 8$ (SD 1.9) \% of total fatty acids; $P<2 \cdot 29 \times 10^{-9}$ ) and the AA: $n$-6-precursor fatty acid ratio, which estimates FADS1 activity (EAm 5.4 (SD 2.2), AfAm 6.9 (SD 2.2); $P=1.44 \times 10^{-5}$ ). In all, seven SNP mapping to the FADS locus revealed strong association with AA, EPA and dihomo- $\gamma$-linolenic acid (DGLA) in the EAm. Importantly, EAm homozygous for the minor allele (T) had significantly lower AA levels (TT 6.3 (SD 1.0); GG 8.5 (SD 2.1); $P=3.0 \times 10^{-5}$ ) and AA:DGLA ratios (TT 3.4 (SD 0.8), GG 6.5 (sD 2.3 ); $P=2.2 \times 10^{-7}$ ) but higher DGLA levels (TT $1.9(\mathrm{SD} 0.4)$, GG $1.4(\mathrm{SD} 0.4) ; P=3.3 \times 10^{-7}$ ) compared with those homozygous for the major allele (GG). Allele frequency patterns suggest that the GG genotype at rs174537 (associated with higher circulating levels of AA) is much higher in AfAm (0.81) compared with EAm (0.46). Similarly, marked differences in rs174537 genotypic frequencies were observed in HapMap populations. These data suggest that there are probably important differences in the capacity of different populations to synthesise LC-PUFA. These differences may provide a genetic mechanism contributing to health disparities between populations of African and European descent.

Key words: SNP: Fatty acid desaturase: Arachidonic acid synthesis

Several lines of evidence indicate that a disproportionate burden of preventable disease, death and disability exists in racial and ethnic minority populations, especially African Americans in the USA. Differences in the prevalence of the metabolic syndrome have been noted in the US National
Health and Nutrition Examination Survey (NHANES) studies, with prevalence notably higher among African American women $^{(1)}$. In addition, the profile of the metabolic syndrome differs among ethnicities, with African Americans showing a smaller contribution of dyslipidaemia (i.e. fewer HDL-cholesterol and

Abbreviations: AA, arachidonic acid; DGLA, dihomo- $\boldsymbol{\gamma}$-linolenic acid; DHS, Diabetes Heart Study; FADS, fatty acid desaturase; GLA, $\boldsymbol{\gamma}$-linolenic acid; LA, linoleic acid; LC-PUFA, long-chain PUFA; LD, linkage disequilibrium.

*Corresponding author: Dr Floyd H. Chilton, fax +1 336716 8501, email schilton@wakehealth.edu 
TAG abnormalities) compared with European Americans ${ }^{(2)}$. However, there is greater insulin resistance among African Americans, even during childhood ${ }^{(3)}$. Likewise, CVD risk shows significant racial and ethnic differences, with the highest age-adjusted death rates observed in African Americans ${ }^{(4)}$. Differences in the prevalence and severity of chronic diseases involving inflammation are further corroborated by differences in inflammatory biomarkers, including C-reactive protein ${ }^{(5,6)}$. The striking racial and ethnic differences in the prevalence and/or severity of common diseases is probably explained by a complex combination of environmental, social, cultural or economic factors, and genetic factors are likely to be very important as well ${ }^{(7)}$.

Agricultural and industrial revolutions have increased the quantity and variety of foods but have not necessarily improved the human diet ${ }^{(8,9)}$. In fact, more than $70 \%$ of the energy consumed by humans today in developed countries would not have been available to our hunter-gather ancestors $^{(8)}$. This rapid shift in the type of energy consumed by modern humans appears to have created a sort of malnutrition in developed countries whereby certain nutrients are not well tolerated by our 'hunter-gather' genes. This problem has become a global issue as Western-derived food supplies and practices expand with global trade. The negative impact of the modern diet on health is likewise exported to developing nations.

Humans can synthesise a wide range of fatty acids, but they lack key enzymes ( $\Delta 12$ and $\Delta 15$ desaturases) necessary to synthesise the initial PUFA used in the key PUFA biosynthetic pathway in mammals ${ }^{(10,11)}$. Therefore, linoleic acid $(18: 2 n$ 6; LA) and $\alpha$-linolenic acid $(18: 3 n-3)$ are essential fatty acids $^{(10)}$. Once obtained from the diet, they are converted to long-chain PUFA (LC-PUFA) by the alternate actions of two fatty acid desaturase (FADS) enzymes ( $\Delta 6$ and $\Delta 5$ desaturases encoded for by FADS 2 and FADS1, respectively) and an elongase enzyme that introduce carbon-carbon double bonds and increases chain length by two carbons, respectively ${ }^{(12)}$. Additionally, preformed LC-PUFA such as arachidonic acid (20:4n-6; AA) can also be readily obtained from human diets. AA is found in relatively high concentrations in the meats of grain-fed animals and eggs ${ }^{(8,13)}$.

Once produced, AA and its metabolic products play important roles in orchestrating immunity and inflammation ${ }^{(14-16)}$ via their ability to directly affect normal and pathophysiological responses through: (i) conversion to potent AA-derived bioactive products (including PG, thromboxanes, leukotrienes and lipoxins); and (ii) regulation of cellular receptors $\left(\mathrm{NF} \kappa \mathrm{B}^{(17,18)}, \mathrm{PPAR}^{(19)}\right.$ and sterol regulatory element-binding protein- $\left.1 \mathrm{c}^{(20,21)}\right)$, thereby modulating the expression of many genes that control immune responses (cytokines such as IL-1, IL-6, IL-12 and TNF- $\alpha$; chemokines such as IL-8, macrophage inflammatory protein-1a and monocyte chemotactic protein-1; adhesion molecules such as intercellular adhesion protein and E-selectin; and inducer effector enzymes such as inducible NO synthase and cyclo-oxygenase- $2^{(22)}$ ).

During the 20th century in the USA, there has been a dramatic increase in LA consumption (from an estimated $2.8 \%$ to nearly $8 \%$ of energy $)^{(13)}$ primarily as a result of increased availability of soyabean oil, margarine and poultry ${ }^{(13)}$. In fact, nearly $85 \%$ of total PUFA in a typical Western diet is LA $^{(23)}$. Biochemical studies using stable isotopes largely carried out in subjects of European ancestry indicate that only a small proportion of dietary LA (about $0.2 \%$ ) can be converted to AA in humans ${ }^{(24)}$. However, humans do appear to be able to synthesise sufficient AA or extract AA from the diet to maintain AA status ${ }^{(25)}$. Nevertheless, this low rate of conversion has been assumed to apply to all human populations equally. For example, the Advisory Committee from the American Heart Association has concluded that at least 5 to $10 \%$ of energy from $[n]-6$ PUFAs reduces the risk of CHD relative to lower intakes $^{,(26)}$. This conclusion was based on several assumptions, one of which concluded that 'wide variations in dietary LA (above minimal essential intakes) do not materially alter tissue AA content ${ }^{(26)}$. Yet the literature suggests that high LA-containing diets can reduce the LC-PUFA content of tissues when modelled from per capita food consumption data ${ }^{(13)}$ or by direct analysis of tissues ${ }^{(27)}$

Studies over the past 5 years suggest that there is probably large genetic variability in the rate of conversion of LA to $\mathrm{AA}^{(28-32)}$. Importantly, genetic variants that are associated with higher levels of AA are also associated with elevated levels of markers of systemic inflammation and the incidence of certain inflammatory disorders ${ }^{(31)}$. The present study examines levels of LA and AA, their association with genetic variants in the FADS gene cluster, and the frequency of high-converting genotypes in patients of African and European ancestry with diabetes or the metabolic syndrome. The results of the present study demonstrate that there is a marked increase in AA and in the frequency of alleles that favour AA synthesis in African Americans with diabetes or the metabolic syndrome. The present study reveals that there are probably differential effects of high concentrations of LA in African and European American populations in the USA and caution should be exercised with regard to dietary recommendations that assume $n-6$ PUFA metabolism is uniform in all human populations.

\section{Experimental procedures}

\section{Subjects}

The study population was derived from the Diabetes Heart Study (DHS; $n$ 229) and included European American ( $n$ 166; from eighty-nine families) and African American (sixty-three from thirty-three families) subjects with diabetes or the metabolic syndrome. Methods for ascertainment and recruitment for the DHS have been described previously ${ }^{(33)}$. Briefly, siblings concordant for type 2 diabetes mellitus without renal insufficiency were recruited, along with unaffected siblings. The metabolic syndrome was defined using the standard definition from the Executive Summary of The Third Report of The National Cholesterol Education Program Expert Panel on Detection, Evaluation, And Treatment of High Blood Cholesterol in Adults (Adult Treatment Panel III) ${ }^{(34)}$. The Wake Forest University School of Medicine Institutional Review Board approved study protocols, and all participants provided written informed consent. 


\section{Fatty acid analysis}

Serum was isolated from fasting whole blood samples. Fatty acid methyl esters were prepared ${ }^{(35)}$ in duplicate from serum samples $(100 \mu \mathrm{l})$ in the presence of an internal standard (triheptadecanoin; NuChek Prep, Elysian, MN, USA) as previously described in detail ${ }^{(36)}$. A panel of twenty-three fatty acids was quantified by GC with flame ionisation detection. Individual fatty acids are expressed as percentage of total fatty acids in a sample. For all samples, data peaks on chromatograms were examined to ensure peak quality and consistency of retention times. Fatty acids in samples were identified based on the retention times of methyl ester derivatives of authenticated fatty acid standards. These standards included Supelco 37 Component FAME Mix (Supleco, Bellefonte, PA, USA) and other individual methyl ester derivatives from Supelco (oleate, cis-11-vaccenoate, linoleate, eicosapentaenoate, $n$-3 docosapentaenoate), Cayman Chemicals (Ann Arbor, MI, USA; stearidonate), Matreya (Pleasant Gap, PA, USA; eicosadienoate, dihomo- $\boldsymbol{\gamma}$-linolenoate, arachidonate) and NuChek Prep (cis-eicosatrienoate, docosadienoate, docosatetraenoate, n-6 docosapentaenoate, docosahexaenoate, tricosanoate, tetracosanoate). Product:precursor ratios of circulating fatty acids, an estimate of enzymic activity, were calculated from fatty acid mass data.

For statistical analyses of fatty acid data, normal kernel density estimation (implemented in S-Plus; TIBCO Software Inc., Palo Alto, CA, USA) was used to obtain estimates of the probability density functions. Linear mixed models were used to assess the racial difference in the fatty acids and ratios adjusting for sex and age. Family was treated as a random effect and age, sex and race as fixed effects. Residuals were examined to assess the model assumptions.

\section{Genotyping and tests for association in the Diabetes Heart Study subjects}

Seven SNP mapping to the FADS gene cluster (rs174537, rs102275, rs174546, rs174556, rs1535, rs174576, rs174579) were selected based on previous publications ${ }^{(30,37,38)}$. Genotypes were determined using a Sequenom MassARRAY SNP genotyping system (Sequenom Inc., San Diego, CA, USA) ${ }^{(39)}$. Of the samples, $3.5 \%$ were genotyped in duplicate with $100 \%$ reproducibility across the SNP. Linkage disequilibrium (LD) was assessed by calculating D' and $r^{2}$ within Haploview $^{(40)}$ relying on a set of independent individuals in the data (a random selection of a single individual from each pedigree, $n 33$ and $n 89$ African American and European American subjects, respectively) and haplotype blocks were defined according to the algorithm of Gabriel et $a l .{ }^{(41)}$.

Allele and genotype frequencies for each SNP were calculated from unrelated probands and tested for departure from Hardy-Weinberg equilibrium using a $\chi^{2}$ goodness-of-fit test. Associations between SNP and traits were performed using a series of variance components measured genotype models as implemented in Sequential Oligogenic Linkage Analysis Routines (SOLAR) ${ }^{(42)}$. Significance was evaluated using the likelihood ratio tests based on the correlation structure suggested by the familial relationships. The additive genetic model was the primary model of interest; however, for SNP with less than ten individuals homozygous for the minor allele a dominant model was analysed and all models included age and sex covariates. When necessary, phenotypes included in these analyses were transformed using the natural logarithm.

\section{Allele frequency analysis of global populations}

Publicly available data for ten populations from the International HapMap project (phase III; http://www.hapmap. org) were used to derive allele frequencies for the rs174537 SNP, which was among those that were genotyped in the DHS population. These data include 1046 samples from the following ten populations: Luhya population in Kenya (LWK; $n$ 110), Yoruba population in Nigeria (YRI; $n$ 147), Mexican ancestry in Los Angeles, CA, USA (MEX; $n$ 58), African American population in the Southwest USA (ASW; $n$ 57), Gujarati Indian population in Houston, TX, USA (GIH; $n$ 101), Japanese population in Tokyo (JPT; $n$ 113), Han Chinese population in Beijing (CHB; $n$ 137), Chinese population in Denver, CO, USA (CHD; $n$ 108), Italian population in Tuscany (TSI; $n$ 102) and a European American population in Utah State, USA (CEU; $n$ 113).

\section{Results}

Serum fatty acid profiles differ in Americans of African and European descent

Fig. 1(a)-(d) show the distribution of $n-6$ PUFA in the sera of African American ( $n$ 63, 41.3\% male, aged 61.0 (SD 10.1) years) and European American ( $n$ 166, 42.7\% male, aged 68.2 (SD 10.5) years) adults with diabetes or the metabolic syndrome from the DHS ${ }^{(33)}$. There was a pronounced enhancement in levels of serum AA $\left(P=2 \cdot 29 \times 10^{-9}\right)$ in African American compared with those in European American subjects. In contrast, no differences were observed in the levels of LA, $\boldsymbol{\gamma}$-linolenic acid (GLA) or dihomo- $\boldsymbol{\gamma}$-linolenic acid (DGLA), all precursors of AA. These data suggest that similar levels of LA are ingested by both populations.

Fig. 1(e)-(g) show the ratios of products to precursors (GLA:LA, DGLA:GLA and AA:DGLA) of circulating fatty acids, which are surrogates of actual enzymic activity at each biochemical step. As suggested by fatty acid levels (Fig. 1(a)-(c)), the conversion of LA to GLA (FADS2 activity) and GLA to DGLA (elongase activity) are not different in subjects of African and European descent. However, there appears to be dramatically different conversion rates for DGLA to AA in the two populations through the $\Delta 5$ desaturase (FADS1 activity) step (Fig. 1(g)). African American subjects exhibited a markedly higher AA:DGLA ratio $\left(P=1.44 \times 10^{-5}\right)$ compared with that in European Americans, suggesting a strikingly increased ability of the former to convert medium-chain (less than twenty carbons) PUFA to LC-PUFA. 
(a)

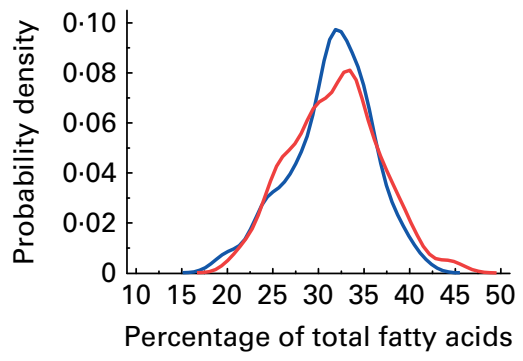

(b)

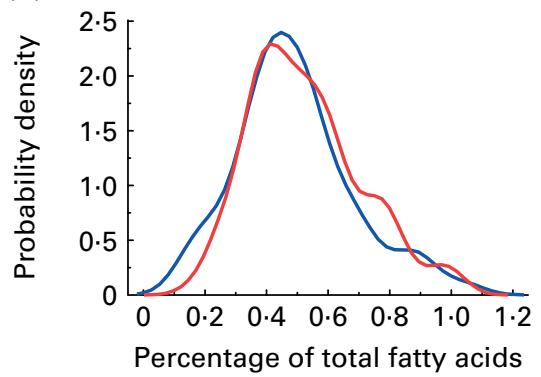

(c)

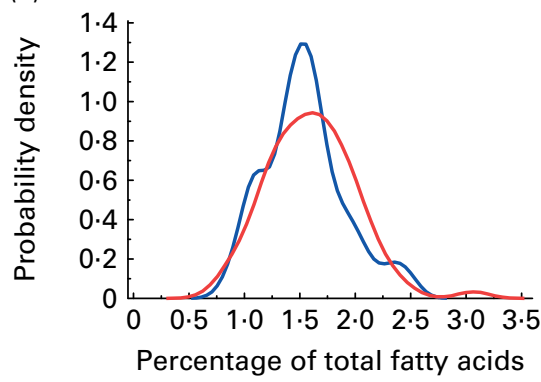

(d)

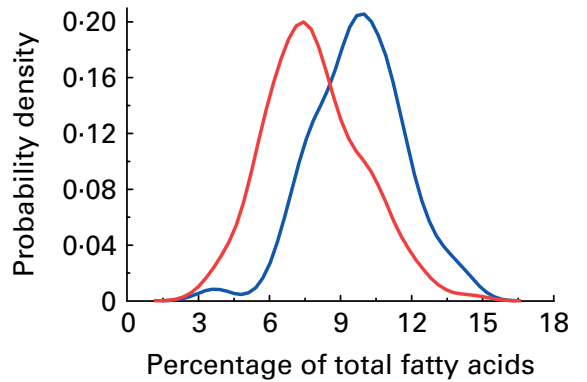

LA

(18:2n-6)

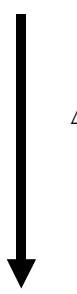

GLA

$(18: 3 n-6)$

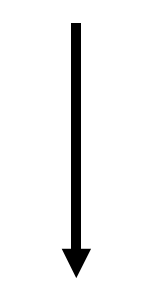

DGLA

$(20: 3 n-6)$

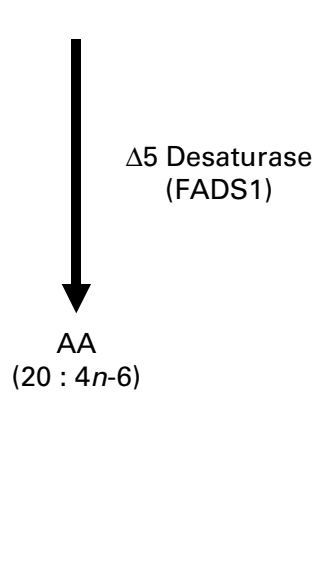

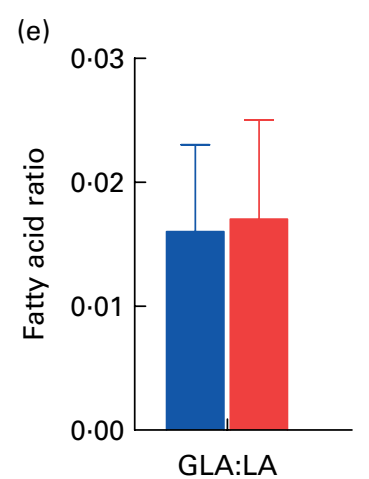

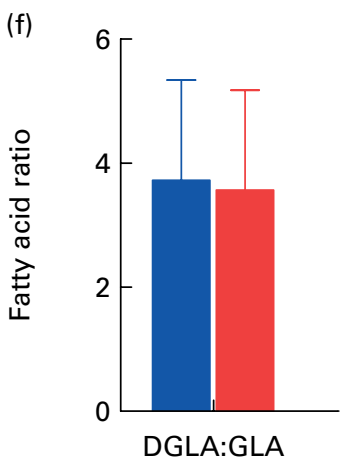

(g)

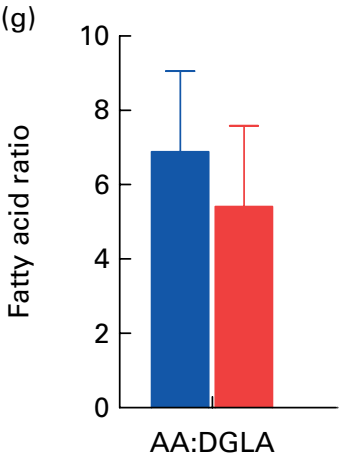

Fig. 1. (a)-(d) Serum fatty acid distributions of $n-6$ PUFA in African American (AfAm; $n 63 ; 41.3 \%$ male; age 61.0 (sD 10.1) years; -) and European American (EAm; $n$ 166; $42.7 \%$ male; age 68.2 (SD 10.5) years; - ) adults with diabetes or the metabolic syndrome from the Diabetes Heart Study. Normal kernel density estimation (implemented in S-Plus; TIBCO Software Inc., Palo Alto, CA, USA) was used to obtain estimates of the probability density functions that show the distribution of subjects having circulating $n$-6 PUFA as a function of percentage of total fatty acids by race. (a), Linoleic acid (LA), $P=0.861 ;$; (b), $\gamma$-linolenic acid (GLA), $P=0.222$; (c), dihomo- $\gamma$-linolenic acid (DGLA), $P=0.534$; (d), arachidonic acid (AA), $P=2.29 \times 10^{-9}$. (e) $-(\mathrm{g})$ The product:precursor ratios of circulating fatty acids were used to estimate (e) fatty acid desaturase 2 (FADS2; GLA:LA ratio; $P=0.348$ ), (f) elongase (DGLA:GLA ratio; $P=0.422$ ) and (g) fatty acid desaturase 1 (FADS1; AA:DGLA ratio; $P=1.44 \times 10^{-5}$ ) enzymic efficiencies for the AfAm $(\square)$ and the EAm ( $\square$ ) populations. Values are means, with standard deviations represented by vertical bars. Linear mixed models were used for statistical analyses to assess the racial difference in the fatty acids and ratios adjusting for sex, age and familial relationships (see Methods).

\section{FADS SNP associations and frequencies}

Tests for association were performed with seven SNP mapping to the FADS gene cluster in DHS subjects. The pattern of association in the European American subjects was highly consistent with previous reports ${ }^{(37,43)}$. Specifically, the pattern in European Americans was in high LD for this region, with a single
LD block $(53 \mathrm{~kb})$ that included the seven SNP across FADS1 and part of FADS2 (not shown). In contrast, no LD blocks were observed in the African Americans (not shown). The strength of association for AA ranged from $1.1 \times 10^{-6}$ to $8.9 \times 10^{-8}$ in the European American subjects (Table 1). Evidence for association was also observed (Table 1) for DGLA $\left(7.5 \times 10^{-6}\right.$ to $\left.7 \cdot 1 \times 10^{-7}\right)$ and GLA $\left(4 \cdot 9 \times 10^{-6}\right.$ to $\left.9.8 \times 10^{-11}\right)$. 
Table 1. Tests of association for seven SNP in the fatty acid desaturase (FADS) loci and $\gamma$-linolenic acid (GLA), dihomo- $\gamma$-linolenic acid (DGLA) and arachidonic acid (AA) in African American ( $n$ 63) and European American ( $n$ 166) subjects from the Diabetes Heart Study

\begin{tabular}{|c|c|c|c|c|c|c|c|}
\hline \multirow[b]{2}{*}{ SNP } & \multirow[b]{2}{*}{ Position } & \multicolumn{3}{|c|}{ African American } & \multicolumn{3}{|c|}{ European American } \\
\hline & & GLA & DGLA & AA & GLA & DGLA & AA \\
\hline rs 174537 & 61309256 & 0.052 & 0.667 & 0.331 & $3.8 \times 10^{-11 * \star \star}$ & $2.4 \times 10^{-7 \star \star \star}$ & $8.9 \times 10^{-8 * \star *}$ \\
\hline rs102275 & 61314379 & 0.087 & 0.682 & 0.058 & $1.6 \times 10^{-10 * \star *}$ & $6.1 \times 10^{-7 \star \star \star}$ & $2.5 \times 10^{-7 \star \star \star}$ \\
\hline rs174546 & 61326406 & 0.027 & 0.877 & 0.693 & $1.7 \times 10^{-10 * \star *}$ & $7 \cdot 1 \times 10^{-7 \star \star \star}$ & $1.1 \times 10^{-6 \star \star \star}$ \\
\hline rs174556 & 61337211 & 0.184 & 0.649 & 0.077 & $5.4 \times 10^{-9 \star \star \star}$ & $7.5 \times 10^{-6 \star \star \star}$ & $1.2 \times 10^{-6 \star \star \star}$ \\
\hline rs1535 & 61354548 & $0.004^{*}$ & 0.828 & 0.167 & $9.8 \times 10^{-11 * \star \star}$ & $6.8 \times 10^{-7 \star \star \star}$ & $7.3 \times 10^{-7 \star \star \star}$ \\
\hline rs174576 & 61360086 & 0.271 & 0.837 & 0.979 & $2.7 \times 10^{-11 * \star *}$ & $5.2 \times 10^{-7 \star \star *}$ & $5.9 \times 10^{-8 * * *}$ \\
\hline rs 174579 & 61362189 & 0.020 & 0.816 & 0.730 & $4.9 \times 10^{-6 * \star *}$ & 0.013 & $0.001^{\star *}$ \\
\hline
\end{tabular}

${ }^{*} P<0.01,{ }^{* *} P<0.001,{ }^{* \star *} P<0.00001$

No associations were observed in the African Americans subjects, which was probably due to the limited sample size of this sample coupled with the much lower allele frequency of the minor alleles. It is important to note that no African American individuals were found in this relatively small population that were homozygous for the minor allele of rs174537.

\section{Genotypic effect of rs174537 on n-6 PUFA and fatty acid} desaturase 1 enzymic efficiency

Fig. 2 shows the distributions of DGLA, AA and the AA:DGLA ratio by genotype at $r \mathbf{1 7 4 5 3 7}$. The major allele $(G)$ is associated with an increase in the mean serum level of AA and is consistent with an additive model in European Americans (Fig. 2(b)). In contrast to the case for AA, the allele (G) was associated with a decrease in mean levels of DGLA, the immediate precursor of AA (Fig. 2(a)), and is also consistent with an additive model in European American subjects. For the ratio of AA:DGLA, an estimate of FADS1 activity (Fig. 2(c)), it was observed that the common allele $(G)$ appeared to be associated with an increased trait mean, i.e. increased enzymic efficiency. As mentioned above, it was not possible to compare levels of PUFA in those with the homozygous minor allele with those with the homozygous major allele as there were no African Americans found in this population that exhibited homozygous minor alleles (Fig. 2(d)-(f)). The mean AA levels in the African American population (GG 9.9 (SD 1.8), GT $9 \cdot 2$ (SD 2.2)) were significantly greater (GG $P=0.003$, GT $P=0.0073$; two-tailed $t$ test) than those in the European American group (GG 8.5 (SD 2.1), GT $7 \cdot 6(\mathrm{SD} \mathrm{1 \cdot 8)})$

\section{FADS rs174537 frequencies differ in populations around the world}

Striking differences were observed in the allele frequencies across a majority of these SNP in the FADS gene cluster between the African American and European American populations in the DHS (Table 2). The resultant genotypic frequencies for rs174537 were skewed toward the homozygous major allele ( $81 \%$ : GG) in the African American population, with only $19 \%$ heterozygous and no homozygous minor allele genotypes observed (Table 2). In contrast, the European
American population exhibited a much lower frequency of the homozygous major allele (46\%), with $43 \%$ heterozygous and $11 \%$ with the homozygous minor allele. To evaluate the genotype distribution of these alleles on a global scale, patterns of genetic variation were examined within the FADS locus, and in particular in rs174537, in populations within The International HapMap Project. Fig. 3 shows striking differences in genotypic frequencies between different populations around the world, with the greatest differences observed between African populations from Kenya (LWK) and Nigeria (YRI) $v$. populations of Mexican ancestry living in Los Angeles, CA, USA (MEX). African Americans in the DHS have genotypic frequencies similar to those in another African American population in the Southwest USA (ASW) and a Gujarati Indian population in Houston, TX, USA (GIH). Greater than $75 \%$ of individuals in each of these populations carry the major allele homozygous GG genotype. In contrast, our DHS European American population had frequencies similar to those of a Japanese population in Tokyo (JPT), a Chinese population in Beijing (CHB), an Italian population in Tuscany (TSI) and a European American population in Utah State, USA (CEU). Less than $50 \%$ of individuals in these populations carry the major allele homozygous GG genotype.

\section{Discussion}

Multi-factorial diseases of chronic inflammation disproportionately affect African Americans in industrialised settings such as the $\mathrm{USA}^{(44)}$, yet appear to be rare in continental Africans. Only $1-2 \%$ of Africans on the African continent have type 2 diabetes, whereas the incidence is $11-13 \%$ in individuals of African descent in industrialised nations consuming a Western $\operatorname{diet}^{(45,46)}$. It is clear that a complex interplay between genes and environment is contributing to these differences.

Several lines of evidence from the present study suggest that this interplay is likely to be important with regard to the dietary consumption and metabolism of $n-6$ PUFA. First, African Americans had higher levels of circulating AA compared with those of European descent. Importantly, there were no differences in levels of the levels of fatty acid precursors (LA and GLA) to $n$ - 6 LC-PUFA in these two populations of patients with diabetes or the metabolic syndrome. To date, few studies have examined the impact of ancestry on LC-PUFA synthesis 
European

American
African

American
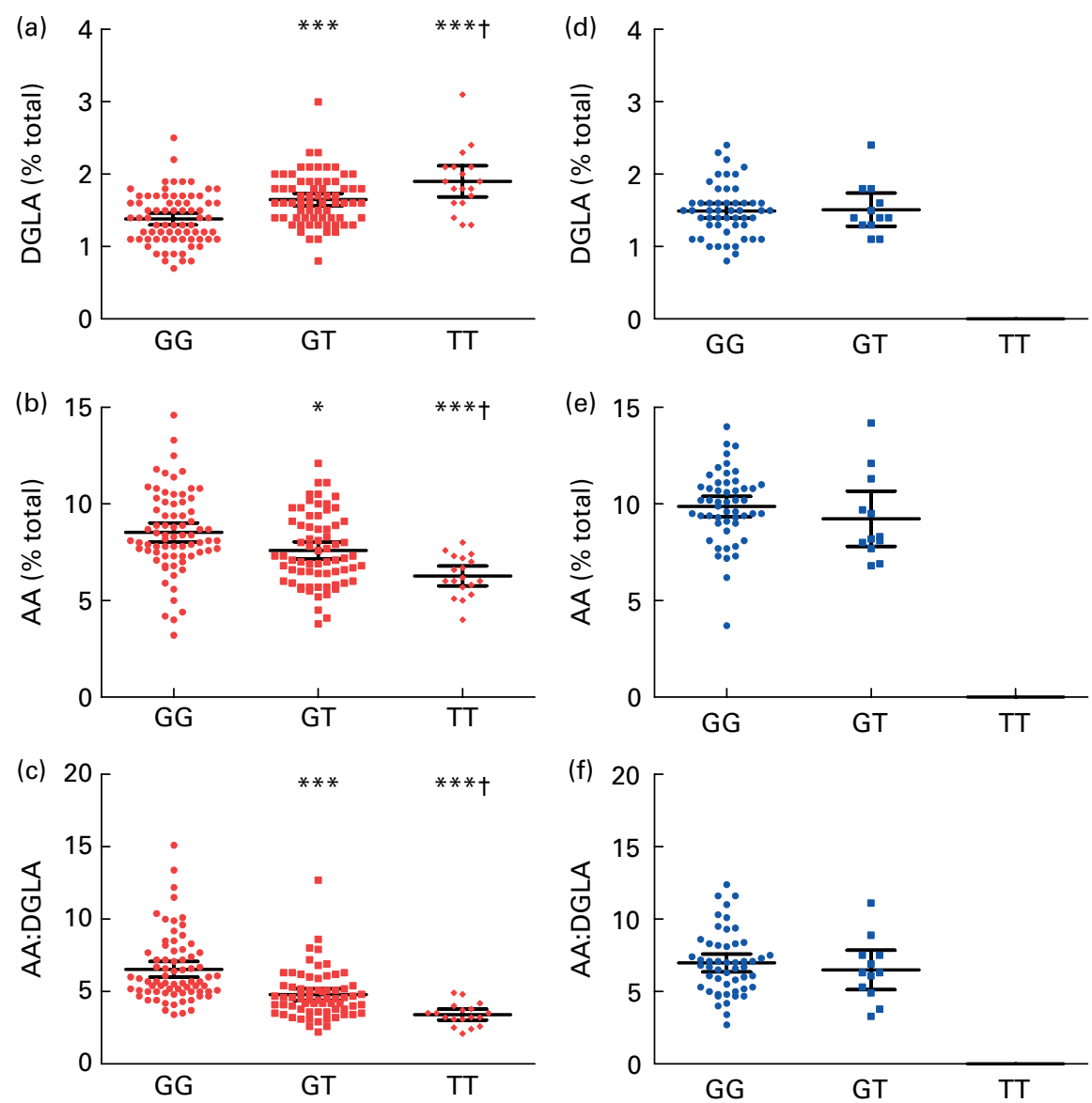

Fig. 2. Fatty acid trait distribution differences between European American ( $n$ 159; (a)-(c)) and African American $(n 63$; (d) - (f)) adults with diabetes or the metabolic syndrome from the Diabetes Heart Study based on genotype at rs 174537. Each sample is represented by a single symbol for European Americans (৫,, , or for African Americans $(\bullet, \square)$ at each genotype. Sample means and $95 \% \mathrm{Cl}$ for the sample mean are shown as the horizontal black line and bars, respectively. Fatty acid data are the percentage of total fatty acids and the arachidonic acid:dihomo- $\gamma$-linolenic acid ratio (AA:DLGA) was calculated from fatty acid mass. Genotypic data were unavailable for seven European American subjects. Mean value was significantly different from that for the $G G$ genotype: ${ }^{*} P<0.01$, ${ }^{* \star *} P<0.00001$ (two-tailed, pair-wise $t$ test within populations). † Mean value was significantly different from that for the GT genotype $(P<0.05$; two-tailed, pair-wise $t$ test within populations).

and levels. In 1991, Horrobin et al. ${ }^{(47)}$ observed that AA levels in plasma phospholipids of nineteen subjects from Zimbabwe Africa were approximately 2 -fold higher than those in a much larger group ( $n$ 458) of subjects with European ancestry. The present study strongly suggests that populations of African ancestry also have higher levels of circulating AA compared with those of European ancestry.

Second, there were marked differences between the African American and European American populations in the present study with regard to frequencies of alleles in several SNP in the FADS gene cluster, which have shown to be important in determining fatty acid levels. Specifically, rs174537 is the SNP near FADS1 that Tanaka et al. ${ }^{(37)}$ have demonstrated to be most associated with AA levels $\left(P=5.95 \times 10^{-46}\right)$. They demonstrated that individuals who were homozygous for the minor allele had significantly lower AA levels compared with those who carried the homozygous major allele. The present study shows that $81 \%$ of African Americans and $46 \%$ of European Americans in the DHS population have the homozygous
GG allele associated with high AA levels. In contrast, no African Americans in the present study population with a homozygous TT allele were found, whereas eighteen out of 159 European Americans carried the homozygous TT allele at rs174537. Additionally, as observed by Tanaka et al. ${ }^{(37)}$, there were significant differences in AA and DGLA as well as the AA:DGLA ratio, an estimate of FAD1 activity, between GG, GT and TT in the European American populations.

Table 2. Genotypic distribution at rs174537 in Diabetes Heart Study populations

\begin{tabular}{|c|c|c|c|c|c|c|}
\hline & \multicolumn{2}{|r|}{$\mathrm{GG}$} & \multicolumn{2}{|r|}{ GT } & \multicolumn{2}{|r|}{ TT } \\
\hline & $n$ & $\begin{array}{l}\text { Genotypic } \\
\text { frequency }\end{array}$ & $n$ & $\begin{array}{l}\text { Genotypic } \\
\text { frequency }\end{array}$ & $n$ & $\begin{array}{l}\text { Genotypic } \\
\text { frequency }\end{array}$ \\
\hline $\begin{array}{l}\text { African } \\
\text { American }\end{array}$ & 51 & 0.81 & 12 & 0.19 & 0 & 0 \\
\hline $\begin{array}{l}\text { European } \\
\text { American }\end{array}$ & 73 & 0.46 & 68 & 0.43 & 18 & 0.11 \\
\hline
\end{tabular}




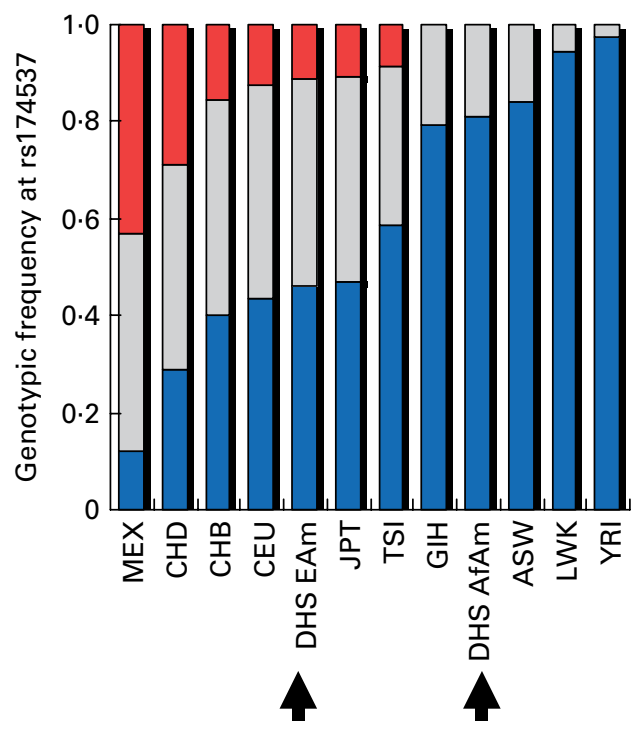

Fig. 3. Distributions of rs174537 genotype frequency in ten HapMap-derived global populations and the Diabetes Heart Study (DHS) African American $($ AfAm) and European American (EAm) subpopulations ( $\uparrow)$, ranked from low to high for the homozygous major allele (GG, $\square$ ). ( $\square$ ), TT; ( $\square$ ), GT; MEX, Mexican ancestry from Los Angeles, CA, USA; CHD, Chinese population in Denver, CO, USA; CHB, Han Chinese population in Beijing; CEU, European American population in Utah State, USA; JPT, Japanese population in Tokyo; TSI, Italian population in Tuscany; GIH, Gujarati Indian population in Houston, TX, USA; ASW, African American population in the Southwest USA; LWK, Luhya population from Kenya; YRI, Yoruba population from Nigeria.

These differences were not seen in the African American population, as the study was not powered to detect such a difference in the small population, which also contained no subjects homozygous for the minor allele in the DHS database. However, a follow-up study has demonstrated comparable (to that in DHS European Americans), highly significant genotypic differences in circulating levels of AA and DGLA and the resultant AA:DGLA ratio in an subset of a larger African American study population (GeneSTAR; Johns Hopkins University, Baltimore, MD, USA) in which the African American sample size was three times that in the present study ${ }^{(48)}$.

Third, an analysis of allele frequencies at rs174537 in HapMap populations around the world shows dramatic differences in allele frequencies among populations. For example, populations from Africa have much higher frequencies of the GG alleles along with very low TT allele frequencies. At the opposite end of the spectrum are endogenous populations from the Americas, which exhibit extremely low frequencies of GG alleles and much higher frequencies of the TT alleles. Other populations (a European population from Tuscany, European Americans from the present study and Utah as well as Japanese and Chinese populations) lie in the middle with regard to allele frequencies of this SNP. If indeed the genotype of this SNP (rs174537) correctly predicts 'efficient converters' (GG), 'modest converters' (GT) and 'nonconverters' (TT) with regard to $\Delta 5$ desaturase (FADS1) enzymic efficiency, then Fig. 3 suggests that a simplistic assumption that wide variations in dietary LA do not alter tissue AA content cannot be made until metabolic studies are carried out in several distinct populations around the world. This is especially important, given that the present paper suggest that the efficiency of LA to AA conversion is probably population dependent.

Finally, numerous studies have demonstrated population differences due to adaptation to pathogens ${ }^{(49)}$, climate and diet $^{(50)}$. However, some past adaptations (such as salt retention and hypertension) are now maladaptive, and can lead to human disease. We propose that this possibility exists for AA and inflammation. Given the elevated levels of $n-6$ medium-chain PUFA (12-17 g/d, principally LA $\left.{ }^{(23)}\right)$ in Western diets, a more efficient capacity to convert medium-chain PUFA to LC-PUFA could promote AA production. This hypothesis is supported by the present study showing that circulating AA in African Americans is on average higher than that in European Americans (Fig. 1), under conditions where genetic backgrounds clearly play a role (Fig. 2). Conditions such as obesity, type 2 diabetes and hypertension are multi-factorial diseases that disproportionately affect African Americans in the $\mathrm{USA}^{(44)}$. Yet, only $1-2 \%$ of Africans on the African continent have type 2 diabetes, whereas the incidence is $11-13 \%$ in individuals of African descent in industrialised nations consuming a Western diet ${ }^{(45,46)}$. There are probably numerous genetic markers and metabolic changes that contribute to these differences, certainly polymorphisms, such as those found in the FADS gene cluster, which could confer increased risk as these populations moved from traditional to Western diets. It is interesting to note that haplotypes of the FADS gene cluster, including variants associated with an elevated AA:LA ratio, are related to both a higher systemic inflammation (as measured by high-sensitivity C-reactive protein) and greater risk of coronary artery disease ${ }^{(31)}$.

For these reasons, recommendations to increase dietary LA levels to 5 to $10 \%$ of dietary energy ${ }^{(26)}$ by organisations such as the American Heart Association are particularly concerning. These recommendations have come largely as a result of registered clinical trials of mixed $n-6+n-3$ PUFA diets and diets in which $n-6+n-3$ PUFA have replaced trans-fatty acids and SFA. Using meta-analyses that took these potential confounders into consideration, Ramsden et al. ${ }^{(51)}$ have observed a potential risk of LA that was probably missed in previous meta-analyses. Five decades of studies and the clinical impact of inhibitors of AA metabolites or metabolism (nonsteroidal anti-inflammatory drugs and leukotriene blockers) support a central role for AA in inflammation. If circulating levels of AA are indeed important, the potential risk of elevating dietary LA would be postulated to differentially affect populations such as African Americans that have a much higher proportion of 'efficient converters' of LA to AA, resulting in higher levels of circulating AA.

\section{Acknowledgements}

The present study was supported by National Institutes of Health (NIH) grants P50 AT002782 (F. H. C.), R01 H1637348 (D. W. B.), R01 NS058700 (D. W. B.), F32DK083214 (C. E. H.), RO1 NR08153 (D. M. B.), RO1 DK 071891 (B. I. F.) and M01 RR000052 (D. M. B.). Additional support was received from 
Wake Forest School of Medicine General Clinical Research Center M01 RR07122 (D. W. B.) and the Wake Forest School of Medicine Center for Public Health Genomics. The authors thank the DHS coordinators, Carrie Smith and Pamela Hicks for their help with obtaining specimens; and Dr R. Mathias for access to study results before publication.

F. H. C. has published books with Rodale and Simon and Schuster and is a founder and consultant to GeneSmart Health, Inc., which may be partially related to his research. These potential conflicts of interest have been disclosed to Wake Forest School of Medicine and to outside sponsors and are institutionally managed. No other authors have a conflict of interest.

C. E. H. performed SNP genotyping and association analyses; M. E. R. selected SNP and designed the genotyping assays; S. S. and P. I. performed fatty acid analyses; H. C. A. performed analyses on the HapMap data; J. T. Z., L. D. C., C. D. L. and D. V. provided statistical and genetic analyses; D. W. B. and B. I. F provided access to DHS data; R. A. M. and F. H. C. generated the hypotheses and designed the experiments; S. S., R. A. M. and F. H. C. prepared the manuscript.

\section{References}

1. Barnes KC, Grant AV, Hansel NN, et al. (2007) African Americans with asthma: genetic insights. Proc Am Thorac Soc 4, $58-68$.

2. Olshansky SJ, Passaro DJ, Hershow RC, et al. (2005) A potential decline in life expectancy in the United States in the 21st century. $N$ Engl J Med 352, 1138-1145.

3. Vaidya D, Szklo M, Ding J, et al. (2007) Agreement of two metabolic syndrome definitions and their association with subclinical atherosclerosis: multi-ethnic study of atherosclerosis cross sectional study. Metab Syndr Relat Disord 5, 343-352.

4. Burns SF, Lee SJ \& Arslanian SA (2009) In vivo insulin sensitivity and lipoprotein particle size and concentration in black and white children. Diabetes Care 32, 2087-2093.

5. Bjorksten B, Clayton T, Ellwood P, et al. (2008) Worldwide time trends for symptoms of rhinitis and conjunctivitis: Phase III of the International Study of Asthma and Allergies in Childhood. Pediatr Allergy Immunol 19, 110-124.

6. Khera A, McGuire DK, Murphy SA, et al. (2005) Race and gender differences in C-reactive protein levels. J Am Coll Cardiol 46, 464-469.

7. Albert MA, Glynn RJ, Buring J, et al. (2004) C-reactive protein levels among women of various ethnic groups living in the United States (from the Women's Health Study). Am J Cardiol 93, 1238-1242.

8. Cordain L, Eaton SB, Sebastian A, et al. (2005) Origins and evolution of the Western diet: health implications for the 21st century. Am J Clin Nutr 81, 341-354.

9. Flinn MV, Geary DC \& Ward CV (2005) Ecological dominance, social competition, and coalitionary arms races: why humans evolved extraordinary intelligence. Evol Hum Behav 26, 10-46.

10. Innis SM (2003) Perinatal biochemistry and physiology of long-chain polyunsaturated fatty acids. I Pediatr $\mathbf{1 4 3}$ Suppl. 4, S1-S8.

11. Innis SM (2008) Dietary omega 3 fatty acids and the developing brain. Brain Res 1237, 35-43.
12. Guillou H, Zadravec D, Martin PGP, et al. (2010) The key roles of elongases and desaturases in mammalian fatty acid metabolism: insights from transgenic mice. Prog Lipid Res 49, 186-199.

13. Blasbalg TL, Hibbeln JR, Ramsden CE, et al. (2011) Changes in consumption of omega- 3 and omega- 6 fatty acids in the United States during the 20th century. Am J Clin Nutr 93, 950-962.

14. Schmitz G \& Ecker J (2008) The opposing effects of $n-3$ and n-6 fatty acids. Prog Lipid Res 47, 147-155.

15. Simopoulos AP (2008) The importance of the omega-6/ omega-3 fatty acid ratio in cardiovascular disease and other chronic diseases. Exper Biol Med 233, 674-688.

16. Torrejon C, Jung UJ \& Deckelbaum RJ (2007) n-3 Fatty acids and cardiovascular disease: actions and molecular mechanisms. Prostaglandins Leukot Essent Fatty Acids 77, 319-326.

17. Novak TE, Babcock TA, Jho DH, et al. (2003) NF-кB inhibition by omega -3 fatty acids modulates LPS-stimulated macrophage TNF- $\alpha$ transcription. Am J Physiol Lung Cell Mol Physiol 284, L84-L89.

18. Soberman RJ \& Christmas P (2003) The organization and consequences of eicosanoid signaling. J Clin Invest 111, $1107-1113$

19. Jump DB \& Clarke SD (1999) Regulation of gene expression by dietary fat. Ann Rev Nutr 19, 63-90.

20. Xu J, Nakamura MT, Cho HP, et al. (1999) Sterol regulatory element binding protein-1 expression is suppressed by dietary polyunsaturated fatty acids. $J$ Biol Chem 274, 23577-23583.

21. Worgall TS, Sturley SL, Seo T, et al. (1998) Polyunsaturated fatty acids decrease expression of promoters with sterol regulatory elements by decreasing levels of mature sterol regulatory element-binding protein. J Biol Chem 273, $25537-25540$

22. Ghosh S \& Karin M (2002) Missing pieces in the NF-кB puzzle. Cell 109, S81-S96.

23. Muskiet FAJ (2010) Pathophysiology and evolutionary aspects of dietary fats and long-chain polyunsaturated fatty acids across the life cycle. In Fat Detection: Taste, Texture, and Post Ingestive Effects [JP Montmayeur and J le Coutre, editors]. Boca Raton, FL: CRC Press. http://www.ncbi.nlm. nih.gov/books/NBK53557/.

24. Hussein N, Ah-Sing E, Wilkinson P, et al. (2005) Long-chain conversion of $\left[{ }^{13} \mathrm{C}\right]$ linoleic acid and $\alpha$-linolenic acid in response to marked changes in their dietary intake in men. J Lipid Res 46, 269-280.

25. Vlaardingerbroek H, Hornstra G, de Koning TJ, et al. (2006) Essential polyunsaturated fatty acids in plasma and erythrocytes of children with inborn errors of amino acid metabolism. Mol Genet Metab 88, 159-165.

26. Harris WS, Mozaffarian D, Rimm E, et al. (2009) Omega-6 fatty acids and risk for cardiovascular disease: a science advisory from the American Heart Association Nutrition Subcommittee of the Council on Nutrition, Physical Activity, and Metabolism; Council on Cardiovascular Nursing; and Council on Epidemiology and Prevention. Circulation 119, 902-907.

27. Friesen RW \& Innis SM (2010) Linoleic acid is associated with lower long-chain $n-6$ and $n-3$ fatty acids in red blood cell lipids of Canadian pregnant women. Am J Clin Nutr 91, 23-31.

28. Malerba G, Schaeffer L, Xumerle L, et al. (2008) SNPs of the FADS gene cluster are associated with polyunsaturated fatty acids in a cohort of patients with cardiovascular disease. Lipids 43, 289-299.

29. Xie L \& Innis SM (2008) Genetic variants of the FADS1 FADS2 gene cluster are associated with altered (n-6) and 
(n-3) essential fatty acids in plasma and erythrocyte phospholipids in women during pregnancy and in breast milk during lactation. J Nutr 138, 2222-2228.

30. Schaeffer L, Gohlke H, Muller M, et al. (2006) Common genetic variants of the FADS1 FADS2 gene cluster and their reconstructed haplotypes are associated with the fatty acid composition in phospholipids. Hum Mol Genet 15, $1745-1756$

31. Martinelli N, Girelli D, Malerba G, et al. (2008) FADS genotypes and desaturase activity estimated by the ratio of arachidonic acid to linoleic acid are associated with inflammation and coronary artery disease. Am J Clin Nutr 88, 941-949.

32. Rzehak P, Heinrich J, Klopp N, et al. (2009) Evidence for an association between genetic variants of the fatty acid desaturase 1 fatty acid desaturase 2 (FADS1 FADS2) gene cluster and the fatty acid composition of erythrocyte membranes. Br J Nutr 101, 20-26.

33. Bowden DW, Lehtinen AB, Ziegler JT, et al. (2008) Genetic epidemiology of subclinical cardiovascular disease in the Diabetes Heart Study. Ann Hum Genet 72, 598-610.

34. Expert Panel on Detection, Evaluation, and Treatment of High Blood Cholesterol in Adults (Adult Treatment Panel III) (2001) Executive Summary of the Third Report of the National Cholesterol Education Program (NCEP). JAMA 285, 2486-2497.

35. Metcalfe LD, Schmitz AA \& Pelka JR (1966) Rapid preparation of fatty acid esters from lipids for gas chromatographic analysis. Anal Chem 38, 514-515.

36. Weaver KL, Ivester P, Seeds MC, et al. (2009) Effect of dietary fatty acids on inflammatory gene expression in healthy humans. J Biol Chem 284, 15400-15407.

37. Tanaka T, Shen J, Abecasis GR, et al. (2009) Genome-wide association study of plasma polyunsaturated fatty acids in the InCHIANTI study. PLoS Genet 5, e1000338.

38. Allayee H, Baylin A, Hartiala J, et al. (2008) Nutrigenetic association of the 5-lipoxygenase gene with myocardial infarction. Am J Clin Nutr 88, 934-940.

39. Buetow KH, Edmonson M, MacDonald R, et al. (2001) High-throughput development and characterization of a genomewide collection of gene-based single nucleotide polymorphism markers by chip-based matrix-assisted laser desorption/ionization time-of-flight mass spectrometry Proc Natl Acad Sci U S A 98, 581-584.

40. Barrett JC, Fry B, Maller J, et al. (2005) Haploview: analysis and visualization of LD and haplotype maps. Bioinformatics 21, 263-265.

41. Gabriel SB, Schaffner SF, Nguyen H, et al. (2002) The structure of haplotype blocks in the human genome. Science 296, 2225-2229.

42. Almasy L \& Blangero J (1998) Multipoint quantitative-trait linkage analysis in general pedigrees. Am J Hum Genet $\mathbf{6 2}$, 1198-1211.

43. Lattka E, Illig T, Heinrich J, et al. (2009) FADS gene cluster polymorphisms: important modulators of fatty acid levels and their impact on atopic diseases. J Nutrigenet Nutrigenomics 2, 119-128.

44. American Heart Association (2009) American Heart Association. Heart Disease and Stroke Statistics - 2008 Update. http://www.americanheartorg/downloadable/heart/12000820 05246HS_Stats\%202008.final.pdf (accessed November 2009).

45. World Health Organization (2009) Diabetes Programme: Facts and Figures. http://www.whoint/diabetes/facts/en/ index.html (accessed November 2009).

46. American Diabetes Association (2009) Diabetes Statistics 2007. http://www.diabetes.org/diabetes-basics/diabetes-statistics/ (accessed November 2009).

47. Horrobin DF, Ells K, Morse-Fisher N, et al. (1991) Fatty acid distribution in plasma phospholipids in normal individuals from different geographical locations. J Nutr Environ Med 2, 249-258.

48. Mathias RA, Sergeant S, Ruczinski I, et al. (2011) The impact of FADS genetic variants on omega- 6 polyunsaturated fatty acid metabolism in African Americans. BMC Genet 12, 50.

49. Haldane JBS (1949) The rate of mutation of human genes. Proc Eighth Int Congr Genet Hered 35, 267-273.

50. Luca F, Perry GH \& Di Rienzo A (2010) Evolutionary adaptations to dietary changes. Ann Rev Nutr 30, 291-314.

51. Ramsden CE, Hibbeln JR, Majchrzak SF, et al. (2010) n-6 Fatty acid-specific and mixed polyunsaturate dietary interventions have different effects on CHD risk: a meta-analysis of randomised controlled trials. Br J Nutr 104, 1586-1600. 